\title{
Inhibition of RhoA expression by adenovirus-mediated siRNA combined with TNF- $\alpha$ induced apoptosis of hepatocarcinoma cells ${ }^{1}$
}

\author{
Kun Yin a, Guihua Zhao ${ }^{\mathrm{a}}$, Xiaodan Huang ${ }^{\mathrm{a}}$, Ge Gao ${ }^{\mathrm{a}}$, Hui Sun ${ }^{\mathrm{a}}$, Qingkuan Wei ${ }^{\mathrm{a}}$, Qiaoqiao Liu ${ }^{\mathrm{a}}$, \\ Mian $\mathrm{Li}^{\mathrm{a}}$, Chao $\mathrm{Xu}^{\mathrm{a}}$, Song $\mathrm{Zhu}^{\mathrm{a}}$, Zaihua $\mathrm{Ba}^{\mathrm{b}}$ and Ge $\mathrm{Yan}^{\mathrm{a}, *}$ \\ ${ }^{a}$ Shandong Academy of Medical Sciences, Shandong Institute of Parasitical Disease, 11 Taibai Middle \\ Road, Jining 272033, China \\ ${ }^{b}$ Jining Medical University, 16 Hehua Road, Jining 272000, China
}

\begin{abstract}
Tumor necrosis factor-alpha (TNF- $\alpha$ ) has been used as an effective treatment for Hepatocellular Carcinoma, however, inducing tumor cell apoptosis by TNF- $\alpha$ alone is still unsatisfactory. RhoA is highly expressed in hepatocarcinoma cells and can be activated by TNF- $\alpha$. The activation of RhoA directly leads to a poor prognosis of HCC. Therefore, we propose to investigate the therapeutic effect of TNF- $\alpha$ together with RhoA siRNA. RhoA inhibition was accomplished by constructing a recombinant adenovirus that can efficiently express RhoA siRNA in HepG2 cells. The recombinant adenovirus AdshRNA-RhoA and AdU6-control were generated by adenovirus-mediated siRNA expression system. The inhibition effects were detected by RT-PCR in addition to immunoblot to quantify the decreased levels of RhoA expression, and the therapeutic effect for HCC was demonstrated by the proliferation and apoptosis ratios of HepG2 cells. The inhibition effects of RhoA by AdshRNA-RhoA were significant at both mRNA and protein levels: the transcription of RhoA mRNA decreased by $74.46 \%$, and the expression of protein decreased by $76.48 \%$. The proliferation rate of HepG2 cells detected by MTT showed that a treatment of AdshRNA-RhoA and TNF- $\alpha$ together could strengthen the suppression ability of TNF- $\alpha$ to HepG2 cells, resulting in approximately $14.2 \%$ more than those treated with only TNF- $\alpha$. FCA and TUNEL assays results revealed that the combined treatment can induce apoptosis in approximately $52.14 \%-65 \%$ of the HepG2 cells, whereas this ratio in the TNF- $\alpha$-alone group was only $21.91 \%-32 \%$. Our results showed that AdshRNA-RhoA can efficiently enhance the TNF- $\alpha$-induced apoptosis of hepatocarcinoma cells. This method might be a useful therapeutic route in HCC and other tumors.
\end{abstract}

Keywords: RhoA, tumor necrosis factor-alpha, adenovirus-mediated siRNA, hepatocarcinoma cells, apoptosis

\footnotetext{
${ }^{1}$ Kun Yin and Guihua Zhao contributed equally to this work.

* Address for correspondence: Ge Yan, Shandong Academy of Medical Sciences, Shandong Institute of Parasitical Disease, 11 Taibai Middle Road, Jining 272033, China. Tel.: +86-0537-2355019; Fax: +86-0537-2353277; E-mail: yange1965@163.com.
}

0959-2989/15/\$35.00 @ 2015 - IOS Press and the authors. 


\section{Introduction}

Hepatocellular carcinoma (HCC) is one of the deadliest human malignancies, accompanied with a very poor prognosis. TNF- $\alpha$ is a pleiotropic proinflammatory cytokine that is primarily produced by macrophages. The main functions of TNF- $\alpha$ include inducing tumor cell apoptosis, enhancing immun-ocyte differentiation, and increasing the permeability of vascular endothelial cells to target tumor neovessels [1-3]. Moreover, TNF- $\alpha$ has also been reported to enhance the therapeutic effects of antitumor drugs. For example, W. Cao, et al. [4] demonstrated that TNF- $\alpha$ promoted doxorubicin-induced cell death and anti-cancer effects via the downregulation of p21 in p53-deficient tumor cells. In addition, the delivery of low TNF- $\alpha$ doses to tumor vasculature can increase the efficacy of chemotherapeutic drugs, such as doxorubicin, melphalan, cisplatin, paclitaxel, and gemcitabine, without an increase in drug cytotoxicity [5]. However, the application of TNF- $\alpha$ has certain limitations, including its toxicity [6] and unsatisfactory effects on tumor cell apoptosis [7], which is correlated to the geranylgeranylation of RhoA [8].

RhoA belongs to the Rho family of GTPases, and high RhoA protein levels and increased activity have been reported in HCC patients [9]. Activating the RhoA/Rho pathway increases tumor cell migratory properties, such as invadopodia, amoeboid migration, and plasticity abilities [10]. Additionally, the expression level of RhoA is directly correlated to the clinicopathologic outcome, as the overexpression of RhoA has been shown to be directly correlated to a poor prognosis of HCC [11]. Furthermore, X.L. Deng, et al. [12] reported that RhoA activation and expression could be induced by TNF- $\alpha$ in mouse cerebral microvascular endothelial cells, and p115RhoGEF may play a role in this process. Thus, this suggests that RhoA activation may be a mechanism of TNF- $\alpha$-induced endothelial cell barrier function. The regulatory effects of TNF- $\alpha$ are modulated at very sensitive levels; for instance, picomolar concentrations of TNF- $\alpha$ stimulated the rapid translocation of RhoA to the plasma membrane, which in turn enhanced monocyte chemotaxis, adhesion, and transmigration activities [13]. Therefore, we presumed that the inhibition of RhoA may be a useful strategy for improving the therapeutic effect of TNF- $\alpha$ on HCC.

In this study, the synergetic effect of TNF- $\alpha$ and adenovirus-mediated RhoA siRNA (AdshRNARhoA) on promoting the apoptosis of hepatocarcinoma cells was investigated. The results demonstrated that the expression level of RhoA in hepatocarcinoma cells was significantly inhibited by AdshRNA-RhoA, and the apoptosis rate of cells that had been treated with AdshRNA-RhoA and TNF- $\alpha$ together was higher than that of cells that had only been treated with TNF- $\alpha$. Our results indicate that RhoA siRNA combined with TNF- $\alpha$ is a potential therapeutic approach for HCC.

\section{Material and methods}

\section{1. siRNAs, plasmids, strains and cell lines}

Three pairs of oligonucleotides targeting three different regions of RhoA (GenBank ID: 387) were designed according to the guidelines proposed by T. Tuschl [14]. siRNA-1 (position 430-448): sense:

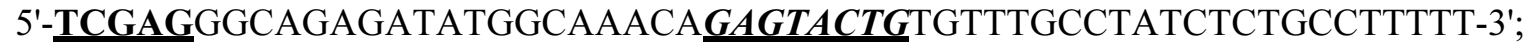
antisense:5'-CTAGAAAAAGGCAGAGATATGGCAAACACAGTACTCTGTTTGCCTATCTCTG CCC- 3'; siRNA-2 (position 226-246): sense:5'-TCGAGGACACCGATGTTATATTGATG $\underline{\text { GTACTG }}$ CATCAATATAACATCGGTGTCTTTTT-3'; antisense: 5'-CTAGAAAAAGACACCGATGTTATA TTGATG $\boldsymbol{C A G T A C T C C A T C A A T A T A A C A T C G G T G T C C}$-3'. siRNA-3 (position 123-144): sense:5'TCGAGCTATGTGGCGGATATCGAGGT GAGTACTGACCTCGATATCCGCCACATAGTTTTT- 
3'; antisense:5'-CTAGAAAAACTATGTGGCGGATATCGAGGTCAGTACTCACCTCGATATCC GCCACATAGC-3'. The underlined sequences are $X b a$ I and Xho I digestion sites and hairpin structures. The plasmids pTZU6+1, pAdTrack, pAdEasy-1 and the E. coli BJ5183, JM109, DH5a strains were preserved in our lab. The HepG2, AD293, and SMMC-7721 cell lines were purchased from the IBCB, CAS.

\subsection{Construction of pTZU6+1-siRNA-RhoA}

The six oligodeoxyribonucleotides were annealed. The methods for constructing the pTZU6+1siRNA-RhoA, extracting the recombinant plasmids and identifying the constructs were the same as those found in our previously published article [15].

\subsection{Cell culture conditions}

AD293, HepG2 and SMMC-7721 cell lines were incubated in RPMI-1640 supplemented with 10\% fetal bovine serum (FBS) at $37^{\circ} \mathrm{C}$, with $100 \mathrm{U} / \mathrm{mL}$ penicillin and $100 \mathrm{U} / \mathrm{mL}$ streptomycin in a $5 \% \mathrm{CO}_{2}$ atmosphere. The cell growth state was observed periodically, and cells in the exponential phase were digested with a $0.25 \%$ trypsin solution containing $0.02 \%$ EDTA.

\subsection{In vitro transfection}

To plasmids, HepG2 cells were transfected respectively with $4 \mu \mathrm{g}$ of pTZU6 +1 , psiRNA-RhoA-1, psiRNA-RhoA-2, or psiRNA-RhoA-3 according to our previous study [15]. To adenovirus, HepG2 cells were infected with the recombinant adenovirus suspension for $72 \mathrm{~h}$ with the titer of $2.0 \times 10^{8}$ $\mathrm{pfu} / \mathrm{mL}$. The infection rates were measured according to the fluorescence expression. The lysate protein concentrations were detected with a BCA kit (Boster, No. AR0146) according to the manufacturer's instructions.

\subsection{Construction of adenovirus-mediated RhoA siRNA}

The RhoA siRNA fragment was first inserted into the Kpn I and Hind III site of the shuttle vector pAdTrack, and then, it was transfected into AD293 cells by lipofection to generate recombinant adenovirus pAdTrack-RhoA. After linearization with Pme I, the pAdTrack-RhoA was co-transfected with the adenovirus frame vector pAdEasy $^{\mathrm{TM}}-1$ into E. coli BJ5183 to generate the recombinant plasmid pAdshRNA-RhoA. This plasmid was linearized again with Pac I and then transfected into the adenovirus package AD293 cell line for 1-2 weeks for virus formation. Subsequently, the virus, which was named AdshRNA-RhoA, was observed by GFP expression and harvested by freeze-thawing and centrifuging the cells. The recombinant virus purification, amplification, and titer determination were all conducted in a manner similar to that of our previous study [16]. The recombinant virus AdU6control was constructed using the same method, but a siRNA fragment was not inserted.

\subsection{Western blot and RT-PCR analysis}

The processes were similar to that in our previously published article [15].

\subsection{MTT assays}


The infected HepG2 cells were cultured in 96-well plates (103-104 cell/well) in $200 \mu \mathrm{L} /$ well medium at $37^{\circ} \mathrm{C}$ in a $5 \% \mathrm{CO}_{2}$ atmosphere for $72 \mathrm{~h}$. TNF- $\alpha$ was added into the experimental group at a concentration of $1 \mu \mathrm{g} / \mathrm{mL}$, and the cells were incubated for $48 \mathrm{~h}$. MTT liquor $(50 \mu \mathrm{L} /$ well) was added to all of the wells, and the cells were continuously incubated for $3 \mathrm{~h}$. Afterwards, the reaction buffer was replaced by a DMSO solution $(150 \mu \mathrm{L} /$ well $)$, and the plate was placed on the microwell plate oscillator for $10 \mathrm{~min}$. The results were measured by an enzyme-labeled instrument at $570 \mathrm{~nm}$.

\subsection{TdT-mediated UTP end labeling (TUNEL) assays}

HepG2 cells were infected with AdshRNA-RhoA and AdshRNA-RhoA combined with TNF- $\alpha$, respectively. The infected cells were grown for $72 \mathrm{~h}$ on coverslips, TUNEL analysis was performed according to the in situ Cell Death Detection kit instruction (Roche Molecular Biochemicals, Switzerland). Any apoptotic strand breaks were revealed by fluorescence and were photographed with a Photometrics Cool Snap ES. Cells infected with Ad-U6-control served as the blank control.

\subsection{Flow cytometric analysis (FCA)}

HepG2 cells were seeded at a density of $1 \times 10^{6}$ cells/well into 6-well plates in a process that is similar to that in our previously published article [16].

\subsection{Statistical analysis}

The results were analyzed using SPSS18.0 software, and difference between two groups was compared using a student test. P-values less than 0.05 were considered to be significant.

\section{Results}

\subsection{Construction of pAdshRNA-RhoA}

The three pairs of RhoA siRNAs were synthesized, annealed, and constructed into the pTZU6+1 vector, respectively. Successful constructions were confirmed by double digestion and sequencing (data not shown). The RhoA protein expression levels were measured by immunoblot, and the normalized RhoA protein levels of HepG2 cells transfected with pTZU6+1, psiRNA-RhoA-1, psiRNA-RhoA-2, and psiRNA-RhoA-3 were $90.25 \%, 29.36 \%, 19.26 \%$, and $45.37 \%$, respectively, as compared with that of HepG2 cells transfected with Lipofectamine (100.0\%) (Figuer 1A). The results indicate that psiRNA-RhoA2 has the highest inhibition efficiency on the RhoA expression of HepG2 cells, and there was no significant difference between psiRNA-RhoA1 and psiRNA-RhoA2 ( $>0.05)$; however, the inhibition effect of psiRNA-RhoA3 was unsatisfactory and was significantly weaker than either psiRNA-RhoA1 or psiRNA-RhoA2 $(\mathrm{p}<0.01)$. Then, the effects of both psiRNA-RhoA1 and psiRNA-RhoA2 on RhoA mRNA expression were detected by semiquantitative RT-PCR, and the normalized RhoA mRNA levels of HepG2 cells transfected with pTZU6+1, psiRNA-RhoA-1, and psiRNA-RhoA-2 were $90.25 \%, 20.37 \%$, and $21.18 \%$, respectively (Figure 1B). These results further confirm that both psiRNA-RhoA1 and psiRNA-RhoA2 can effectively inhibit RhoA expression, and again, there was not a significant difference between them $(p>0.05)$. Thus, siRNA-2 was selected as the candidate for adenovirus-mediated siRNA construction. 
The pAdshRNA-RhoA construct was generated using the recombinant psiRNA-RhoA-2 and the adenovirus shuttle vector pAdTrack (Figure 1C). Successful construction was identified by restriction digest analysis (Figure 1D) and was confirmed by sequencing. The digested products of pAdTrackRhoA were 50 bp larger than the control group located in lane 4, thereby indicating that the U6+1 promoter and the siRNA inverted repeat sequence fragment were successfully cloned into the vector pAdTrack. The PmeI-linearized positive pAdTrack-RhoA and pAdEasy-1 were co-transfected into E. coli BJ5183 to generate the recombinant pAdshRNA-RhoA. Digestion with PacI yielded a $4.5-\mathrm{kb}$ or 3.0-kb DNA fragment and confirmed the positive recombinant in lanes 3-5 (Figure 1E). The control plasmid pAdU6-control was constructed in the same manner as pAdshRNA-RhoA, except only the U6+1 promoter was cloned into pAdU6-control.

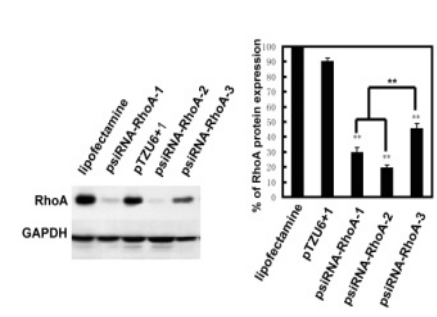

A

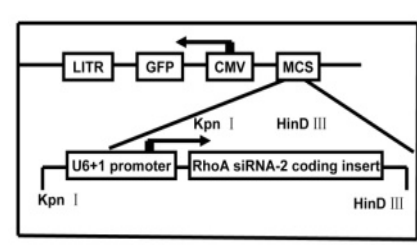

C

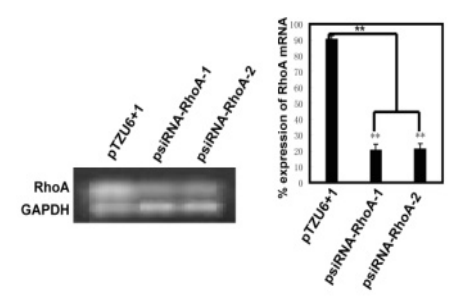

B

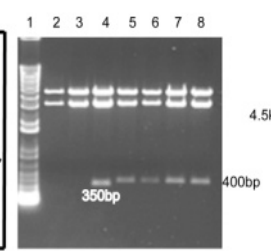

D

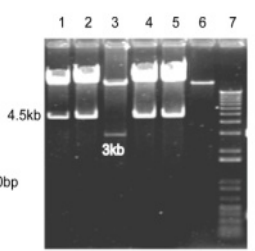

E

Fig. 1. Construction of recombinants pAdshRNA-RhoA and pAdU6-control. A: Left: Western blot analysis of RhoA protein expression in HepG2 cells. Right: Histogram showing quantification of the RhoA bands. B: Left: Semi-quantitative RT-PCR analysis of RhoA mRNA expression in HepG2 cells. Right: Histogram showing quantification of the RT-PCR results. GADPH was used as an internal control. ${ }^{*} p p<0.01$ as compared with control; $\mathrm{n}=3$. C: Schematic outline of recombinant adenovirus vector cloning strategy. The plasmid psiRNA-RhoA-2 was digested by HindIII and KpnI, and the digested fragment, including siRNA-2 and the U6+1 promoter, was inserted into the plasmid pAdTrack to generate pAdTrack-RhoA. D: Restriction analysis of recombinant constructs pAdTrack-RhoA and pAdTrack-U6 with KpnI and HindIII. Lane 1, marker; lanes 2-3, pAdTrack; lane 4, pAdTrack-U6; lanes 5-8, pAdTrack-RhoA. E: Digestion of recombinant pAdshRNARhoA and pAdU6-control with PacI. Lanes 1-2, pAdU6-control; lanes 3-5, pAdshRNA-RhoA; lane 6, pAdEasy-1; lane 7, marker.

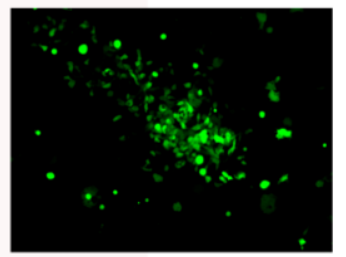

A

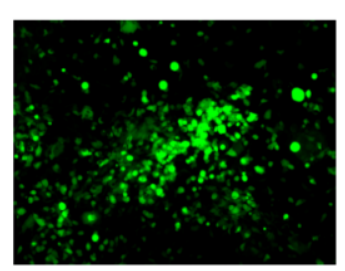

B

Fig. 2. GFP expression in cells infected by recombinant adenovirus. A: AD293 cells were infected with pAdshRNA-RhoA for 7 days. B: AD293 cells were infected with pAdshRNA-RhoA for 12 days. Increased GFP expression was observed. Cells were examined under fluorescence microscopy at $200 \times$ magnification. 


\subsection{Generation of recombinant adenovirus AdshRNA-RhoA}

Since the AD293 cell line allows for the production of infectious virus particles when cells are transfected with E1-deleted adenovirus vectors [17] (such as pAdEasy ${ }^{\mathrm{TM}}-1$ vector), it was used to propagate adenoviral recombinants in this study. The adenoviral recombinants pAdshRNA-RhoA and pAdU6-control were each digested with PacI and then transfected into HepG2 cells. The original virus suspensions were harvested when the infected cells appeared to float and exhibited visible virus spots after 7-12 days. Next, larger numbers of HepG2 cells were infected with the original virus suspensions in order to generate more adenovirus. The virus was collected according to the GFP expression observed by fluorescence microscopy (Figures $2 \mathrm{~A}$ and $2 \mathrm{~B}$ ). The titer of the recombinant virus was as follows: AdshRNA-RhoA, $2.1 \times 10^{8} \mathrm{pfu} / \mathrm{mL}$ and AdU6-control, $1.8 \times 10^{8} \mathrm{pfu} / \mathrm{mL}$. After cesium chloride gradient centrifugation, the final titer was $2.4 \times 10^{9} \mathrm{pfu} / \mathrm{mL}$ for AdshRNA-RhoA and $2.1 \times 10^{9} \mathrm{pfu} / \mathrm{mL}$ for AdU6-control.

\subsection{AdshRNA-RhoA efficiently inhibited RhoA mRNA and protein expression}

The RhoA mRNA expression levels in infected HepG2 cells were examined by semi-quantitative reverse transcription-polymerase chain reaction (RT-PCR). HepG2 cells were infected with AdshRNA-RhoA or AdU6-control for $72 \mathrm{~h}$. It was found that the RhoA levels were significantly weaker in AdshRNA-RhoA-transduced cells than in either uninfected cells or cells infected with AdU6-control $(\mathrm{p}<0.01)$. Quantification of the RT-PCR results by the ImageMaster TotalLab System revealed that the RhoA mRNA expression levels in the AdshRNA-RhoA-infected cells and AdU6control-infected cells were $25.54 \%$ and $99.30 \%$, respectively, as compared to untreated cells $(\mathrm{p}<0.01)$. Accordingly, the levels of the GAPDH internal control remained unchanged (Figure 3A).
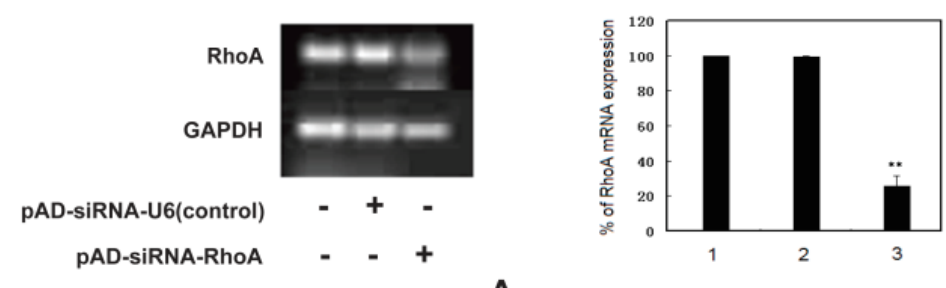

A
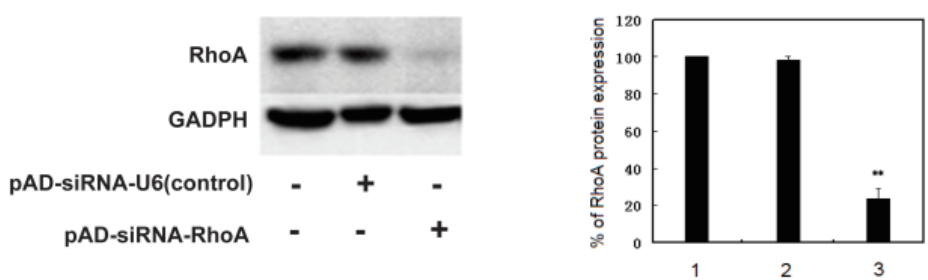

B

Fig. 3. RhoA expression levels in pAdshRNA-RhoA-infected HepG2 cells. A: Left: Semi-quantitative RT-PCR analysis of RhoA mRNA expression in pAdshRNA-RhoA-infected HepG2 cells. Right: Histogram showing quantification of the RTPCR results. B: Left: Western blot analysis of RhoA protein expression in pAdshRNA-RhoA-infected HepG2 cells. Right: Histogram showing quantification of the RhoA bands. The RhoA mRNA and protein levels in the AdsiRNA-RhoA-infected cells were significantly reduced as compared to that of the uninfected control cells or AdU6-control-infected cells. GADPH was used as an internal control. This experiment was repeated twice with similar results. 1, HepG2 cells; 2, HepG2 cells+AdU6-control; 3 , HepG2 cells+AdshRNA-RhoA; ${ }^{*} p<0.01$ as compared with control; $\mathrm{n}=2$. 
Next, western blot analysis was performed to analyze the RhoA protein expression levels after adenoviral infection. The results were similar to the RT-PCR results. More specifically, the AdshRNA-RhoA-infected cells showed significantly reduced levels of RhoA protein expression $(23.52 \%)$ than in either the control cells or the AdU6-control-infected cells $(98.20 \%)(p<0.01)$. Moreover, there was not a significant difference between the RhoA levels in uninfected cells and those of AdU6-control-infected cells ( $p>0.05$ ). Again, the GAPDH expression was unchanged (Figure 3B). These results indicate that the recombinant adenovirus AdshRNA-RhoA efficiently inhibited RhoA expression at both the mRNA and protein levels.

\subsection{Inhibition of HepG2 cell proliferation by TNF- $\alpha$ was augmented with AdshRNA-RhoA}

Because the proliferation activity of a tumor cell is important for its metastasis and invasion ability, an MTT assay was used to evaluate HepG2 cell proliferation activity. The negative control group was infected with the AdshRNA-U6 adenovirus to eliminate any possible interference from the adenovirus- mediated siRNA expression system. The HepG2 cells were divided into three groups and were treated as follows (see Table 1): TNF- $\alpha$, AdshRNA-RhoA, and TNF- $\alpha+$ AdshRNA-RhoA. Surprisingly, the cells that were solely infected with AdsiRNA- RhoA showed no significant difference in cell survival rate as compared to AdU6-control-infected cells $(p>0.01)$, thus indicating that the inhibition of RhoA expression did not directly impact the HepG2 cell proliferation activity. In contrast, a considerably low survival rate of HepG2 cells (47.5\%) was observed in the TNF- $\alpha$ alone treatment group, which demonstrates that TNF- $\alpha$ is an efficient candidate for developing therapeutic treatments for HCC. Moreover, when TNF- $\alpha$-treated HepG2 cells were simultaneously infected with AdshRNA-RhoA, the suppression rate of HepG2 cells increased markedly $(p<0.001)$ as compared to the cells treated by TNF- $\alpha$ alone. For example, the recombinant AdshRNA-RhoA adenovirus improved the suppression effect of TNF- $\alpha$ from $52.5 \%$ to $66.7 \%(\mathrm{p}<0.01)$, demonstrating that adenovirus-mediated RhoA siRNA can effectively augment TNF- $\alpha$ 's effect on the inhibition of HepG2 cell proliferation in vitro.

\subsection{AdshRNA-RhoA enhanced the apoptosis of HepG2 cells induced by TNF- $\alpha$}

Because the ability to induce tumor cell apoptosis is a necessary requirement of antitumor drug design and application, TUNEL and FCA analyses were performed to assess the potential antineoplastic effect of both AdshRNA-RhoA alone and the combined AdshRNA-RhoA + TNF- $\alpha$. Cells infected with Ad-U6-control were used as the experimental control in order to confirm any

Table 1

Statistical data of MTT results

\begin{tabular}{llll}
\hline & $\begin{array}{l}\text { OD Value } \\
( \pm \mathrm{SD}, \mathrm{n}=6)\end{array}$ & $\begin{array}{l}\text { Cell Survival } \\
\text { Rate }(\%)^{1}\end{array}$ & $\begin{array}{l}\text { Suppression } \\
\text { Rate }(\%)^{2,3}\end{array}$ \\
\hline AdshRNA-U6(control) & $0.648 \pm 0.023$ & $100.0 \pm 2.3$ & $0.0 \pm 2.3$ \\
AdshRNA-RhoA & $0.628 \pm 0.014$ & $97.0 \pm 3.9$ & $3.0 \pm 3.9$ \\
TNF- $\alpha$ & $0.307 \pm 0.011$ & $47.5 \pm 1.6$ & $52.5 \pm 1.6^{*}$ \\
AdshRNA-RhoA+TNF- $\alpha$ & $0.216 \pm 0.014$ & $33.3 \pm 1.6$ & $66.7 \pm 1.6^{*}$ \\
\hline
\end{tabular}

Note: ${ }^{1}$ Cell survival rate was calculated by the equation: survival rate $\%=$ experimental group OD value/control group OD value $\times 100 \%$. ${ }^{2}$ Suppression rate was calculated by the equation: suppression rate $\%=1-$ survival rate $\%$.

${ }^{3}$ These data were used to evaluate HepG2 cell proliferation activity as influenced by the different treatments as indicated. A suppression rate of greater than $30 \%$ could be declared as positive for drug susceptibility.

* There was significant difference between the two groups, $\mathrm{p}<0.01$. 
influence from the recombinant adenovirus and empty siRNA expression vector. The other cells were divided into three groups as was done for the MTT assay.

As shown in Figure 4A, the results indicate that the adenovirus-mediated siRNA expression system did not affect the TUNEL assay, as almost all of the infected cells retained their normal morphology and were closely packed on the culture dish. In addition, the AdshRNA-RhoA-infected cells showed similar results to the Ad-U6-control cells, as is shown in Figure 4B, with only a small number of cells (no more than 10\%) stained in the nuclei and lightly stained in the cytoplasm. The majority of the cells maintained normal cell morphology, thus demonstrating that the inhibition of RhoA expression only slightly influences HepG2 cell apoptosis. The difference was not statistically significant $(p>0.05)$.

However, more TUNEL-positive cells were observed in the TNF- $\alpha$ only treatment, as is shown in Figure 4C. Approximately $32 \%$ of the TNF- $\alpha$-treated HepG 2 cells were stained brownish red and displayed karyopyknosis. Moreover, there were some apoptotic differences in comparison to the cells treated by AdsiRNA-RhoA, such as adherent cells also exhibiting cytoplasm staining. However, when cells were treated with TNF- $\alpha$ and AdsiRNA-RhoA together, the majority of the cells exhibited typical apoptotic phenomenon, as is shown in Figure 4D. The TUNEL-positive cells occupied a large area of the observed visual field and floated up in the culture dish. The apoptosis ratio increased to about $65 \%$ (Figure 4E) of approximately 1,000 cells $(\mathrm{p}<0.05)$.

The FCA results showed that the apoptosis rates of the Ad-U6-control group and AdshRNA-RhoA group were only $0.82 \%$ and $2.98 \%$, respectively ( $p>0.05$ ), as shown in Figures $5 \mathrm{~A}$ and $5 \mathrm{~B}$. However, an obvious hypodiploid peak was observed in the TNF- $\alpha$ group (Figure 5C), and the apoptosis ratio is creased to $21.91 \%$. When cells were treated with the combined AdshRNA-RhoA+TNF- $\alpha$, the corresponding peak rose even higher (Figure 5D), and the apoptosis ratio increased to $52.14 \%$, thereby indicating that the combined treatment has the potential ability to induce the apoptosis of HepG2 cells $(\mathrm{P}<0.01)$.

The study's results revealed that RhoA si s not obviously affect HepG2 cell apoptosis,

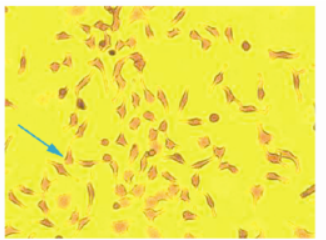

A

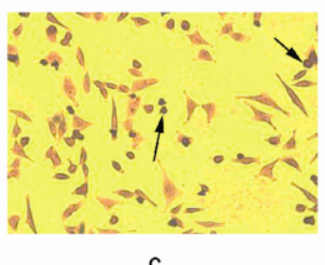

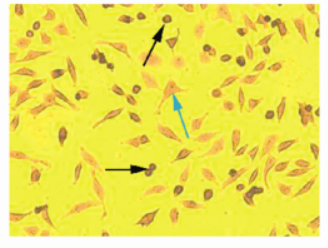

B

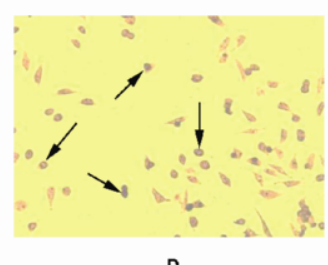

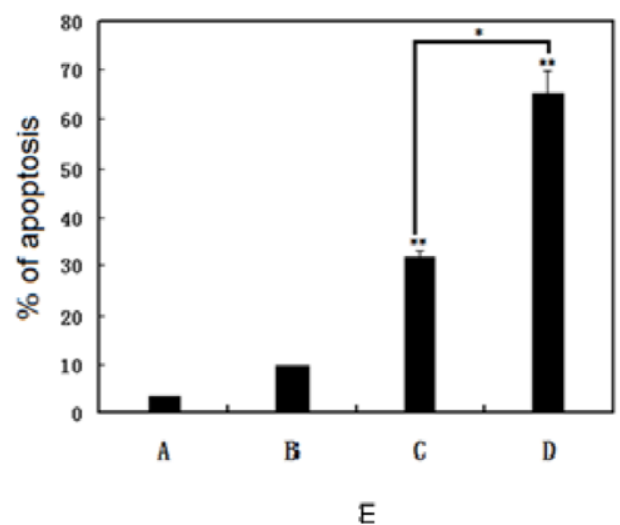

E

Fig. 4. Effects of combined treatment of AdsiRNA-RhoA and TNF- $\alpha$ on the apoptosis of HepG2 cells as determined by TUNEL assay. HepG2 cells were infected with either A: AdU6-control and empty pTZU6+1 vector or B: AdshRNA-RhoA for $72 \mathrm{~h}$. Two additional groups of HepG2 cells were treated with C: only $1 \mu \mathrm{g} / \mathrm{mL} \mathrm{TNF}-\alpha$ or D: TNF- $\alpha$ and AdshRNA-RhoA together for $72 \mathrm{~h}$. TUNEL-positive apoptotic cells were stained brownish red in the nucleus and displayed karyopyknosis, as indicated by black arrows. Blue arrows indicate TUNEL-negative cells. Original magnification: $\times 400$. This experiment was repeated twice with similar results. E: Histogram of quantification of the apoptosis rate in (A-D). The average numbers of HepG2 apoptotic cells were calculated by counting the TUNEL-positive cells in five different visual fields, with 200 cells counted in each field. ${ }^{*} p<0.05$ and ${ }^{*} p<0.01$ as compared with control; $\mathrm{n}=2$. 
and low TNF- $\alpha$ doses are capable of inducing HepG2 cell apoptosis but only to a certain low extent. However, the combined treatment significantly increased the number of apoptotic cells, which indicates that the specific inhibition of RhoA expression by adenovirus-mediated siRNA can enhance the spontaneous apoptosis of HepG2 cells induced by TNF- $\alpha$.

\section{Discussion and conclusion}

Several studies have demonstrated that the inhibition of target genes that are highly expressed in tumor cells is a useful therapeutic route in HCC [18], especially when targeting genes relevant to the combination of unrestrained cell proliferation and impaired apoptosis [19]. Furthermore, RNA interference has been widely used in the anti-tumor field; however, the traditional method of employing siRNA vectors is highly limited. In particular, the transfection efficiency is not stable, and applying the technology to animal model systems is difficult to accomplish $[16,20]$.

In this study, when HepG2 cells were infected with a recombinant virus for $36 \mathrm{~h}$, approximately $90 \%$ of the HepG2 cells displayed GFP expression under fluorescence microscopy, which indicates that the adenovirus had high transduction efficiency in the target tumor cells. It was also found that the infection efficiency is correlated to the cell source. The transfection efficiency of the recombinant virus was evaluated by the MOI (multiplicity of infection) value. The $100 \%$ infection rate of the AdshRNA-RhoA and AdU6-control virus to the HepG2 cell line required the MOI to reach about 20, which is the same value that was necessary for the other human hepatoma cell line SMMC-7721. However, in the human embryonic kidney cell line AD293, the MOI value was 10. Thus, the cell source from different tissues likely plays an important role in regulating the recombinant adenovirus transfection efficiency, and we believe that further research on this point will be highly beneficial for the rational application of recombinant adenovirus. Furthermore, the application of such a stable adenovirus vector system is convenient and does not produce any adverse effects on animal models [21]. Thus, this system can be easily used in animal experiments to test gene therapeutic effects, as was shown in our previously published article [20].

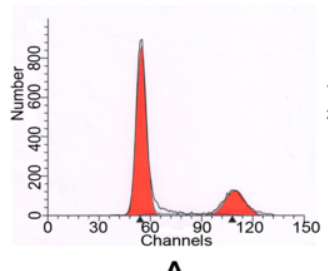

A

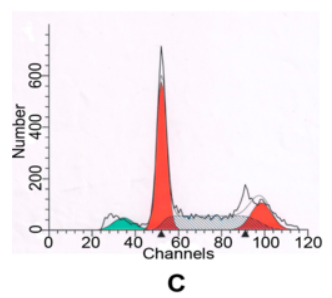

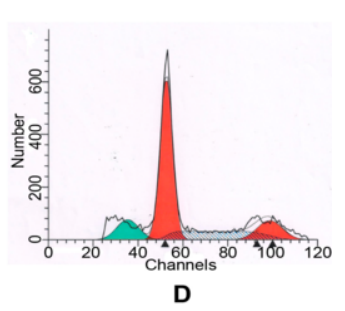

Fig. 5. Apoptotic cells of HepG2 as detected by FCA assay. HepG2 cells were infected with A: AdU6-control and empty pTZU6+1 vector; B: AdshRNA-RhoA for $72 \mathrm{~h}$; C: only $1 \mu \mathrm{g} / \mathrm{mL}$ TNF- $\alpha$; or D: TNF- $\alpha$ and AdshRNA-RhoA together for 72 $\mathrm{h}$. The blue hypodiploid peak in the G0-G1 period represents cell apoptosis. This experiment was repeated twice with similar results. 
Many studies have previously established that the Rho GTPases/Rho-effector pathway plays important roles in mediating HCC metastasis, based on its foremost function of orchestrating the cytoskeletal reorganization in the cell [22]. For example, Wong et al. successfully suppressed the proliferation and invasion of HCC cells by deleting the Rho GTPase-activation protein in the liver [23]. As a member of the Rho GTPase family, RhoA is an important factor in promoting the uncontrolled proliferation as well as the invasive and metastatic properties of cancer cells [24]. Accordingly, the clinicopathological correlation of RhoA levels with HCC has been widely evaluated, and it was found that a high RhoA protein expression is detectable in both idiopathic and early-stage recurrent HCC [9], and high expression is also closely related to venous invasion, differentiation [11], tumor progression, and the metastasis of hepatocarcinoma cells $[25,26]$. Moreover, proteomic analysis also identified RhoA as one of the most significantly up-regulated proteins in HCC [27]. Thus, RhoA was selected as a good target for HCC therapy.

Recently, biological approaches for inhibiting RhoA expression in order to induce tumor cell apoptosis have been tested with relative success [28, 29]. One report demonstrated that the suppression of RhoA expression in HepG2 and Hep3B cells by RNA interference led to cell growth inhibition, apoptosis, and a decrease in migration [27]. However, our results showed that AdshRNA-RhoA produces no apparent effect on either suppressing proliferation or inducing the apoptosis of infected HepG2 cells, despite its confirmed effect in silencing RhoA expression. These results also contrasted with results from our similar investigation in the breast cancer cell line T47D. Moreover, we also tested the suppression effect of AdshRNA-RhoA in this cancer cell line and found that the AdshRNARhoA exhibits intensive inhibition activity on T47D cell proliferation and induced a large portion of cell apoptosis (data not shown). The mechanism of this apparent discrepancy remains unclear; however, a new report on miR-21 [30] may provide some assistance in elucidating this inconsistency. In this new report, it was demonstrated that the suppression of miR-21 by the newly discovered regulator indole-3-carbinol (I3C) could repress the PTEN/AKT (phosphatase and tensin homolog/protein kinase B) pathway, leading to an inhibition of tumorigenesis in the hepatocarcinomal cell. Moreover, activation of RhoA by the downstream effector Rock (RhoA-associated kinase) can stimulate the phospholipid phosphatase activity of PTEN in human embryonic kidney cells and leukocytes [31], and in turn, the activated PTEN down-regulates AKT activity, which is essential for cell proliferation [32]. Furthermore, F. Fusella, et al. [33] also reported that PTEN destabilization caused by the high morgana levels in breast cancer cells triggered the PI3K/AKT survival pathway; thus, the cells' self-protective activity from various apoptotic stimuli was strongly enhanced. Differences in the apoptotic rates among different cancer cell lines caused by RhoA inhibition may be correlated with the RhoA-ROCK-PTEN pathway, but the mechanism needs to be further investigated. We also speculate that RhoA may need an enhancer or cooperator, such as TNF- $\alpha$, for achieving its maximal function on suppressing hepatocarcinoma cell proliferation and inducing apoptosis.

It has been widely established that TNF- $\alpha$ can enhance the function of anti-tumor drugs, and the RhoA/Rho signaling pathway and RhoA activity can both be influenced by TNF- $\alpha$ in diverse cell lines. Furthermore, TNF- $\alpha$ can induce the activation of the ERK/GEF-H1/RhoA pathway in tubular cells, which is mediated through Src- and TACE-dependent EGFR activation [2]. The RhoA mRNA expression in human bronchial smooth muscle can also be induced by cytokines, such as IL-13 and TNF- $\alpha$, and additionally, STAT6 and NF-KB are important for this up-regulation [34]. TNF- $\alpha$ can also induce the up-regulation of RhoA via NF- $\mathrm{KB}$ activation in cultured human bronchial smooth muscle cells [35]. This up-regulation mechanism of RhoA was mediated by GEFF-H1, as the siRNA-induced down-regulation of GEFF-H1 prevented the activation of the Rho pathway [36]. However, the precise candidates involved in the TNF- $\alpha$ and RhoA correlation in tumorigenesis remain elusive. Furthermore, 
it is important to note that, although it has been widely established that TNF- $\alpha$ can enhance the function of anti-tumor drugs, in this study, it appeared that AdshRNA-RhoA rather than TNF- $\alpha$ played the auxiliary role. These results have demonstrated that the down-regulation of RhoA together with TNF- $\alpha$ can significantly induce HepG2 cell apoptosis, which suggests that the suppression of RhoA can improve the auxiliary curative effect of TNF- $\alpha$ on both cell proliferation and apoptosis. The molecular mechanisms underlying these effects are still unclear and need to be further investigated.

In conclusion, the combined RhoA adenovirus-mediated siRNA with low doses of TNF- $\alpha$ in HepG2 cells significantly affects cell apoptosis, as well as affects the inhibition of cell proliferation. This is the first study to use an anti-tumor cytokine and Rho GTPase siRNA together to induce tumor cell apoptosis, and the data we have produced suggest promising applications in the HCC gene therapy field. As both RhoA and TNF- $\alpha$ are relevant in metastasis [1,26, 37], this data may also be applied to the inhibitions of other types of tumor cells.

\section{Acknowledgment}

This work was supported by grants from the National Natural Science Foundation of China (81041075), the Science and Technology Development Program of Shandong Province (2006GG2302011), and the Natural Science Foundation of the Shandong Province (Q2007D04).

\section{References}

[1] F. Balkwill, Tumour necrosis factor and cancer, Nature Reviews Cancer 9(2009), 361-371.

[2] E. Kakiashvili, Q. Dan, M. Vandermeer, Y. Zhang, F. Waheed, M. Pham and K. Szászi, The epidermal growth factor receptor mediates tumor necrosis factor-alpha-induced activation of the ERK/GEF-H1/RhoA pathway in tubular epithelium, Journal of Biological Chemistry 286 (2011), 9268-9279.

[3] C. Emmanuel, M. Huynh, J. Matthews, E. Kelly and H. Zoellner, TNF-alpha and TGF-beta synergistically stimulate elongation of human endothelial cells without transdifferentiation to smooth muscle cell phenotype, Cytokine 61 (2013), $38-40$.

[4] W. Cao, S.L. Ma, J. Tang, J. Shi and Y. Lu, A combined treatment TNF-alpha/doxorubicin alleviates the resistance of MCF-7/Adr cells to cytotoxic treatment, Biochimica et Biophysica Acta 1763 (2006), 182-187.

[5] A. Sacchi, A. Gasparri, C. Gallo-Stampino, S. Toma, F. Curnis andA. Corti, Synergistic antitumor activity of cisplatin, paclitaxel, and gemcitabine with tumor vasculature-targeted tumor necrosis factor-alpha, Clinical Cancer Research 12 (2006), 175-182.

[6] D.R. Spriggs, M.L. Sherman, H. Michie, K.A. Arthur, K. Imamura, D. Wilmore, E. Frei and D.W. Kufe, Recombinant human tumor necrosis factor administered as a 24-hour intravenous infusion, A phase I and pharmacologic study, Journal of the National Cancer Institute 80 (1988), 1039-1044.

[7] J. Li, R. Zheng and Z. Wang, Mechanisms of the induction of apoptosis in human hepatoma cells by tumour necrosis factor-alpha, Cell Biology International 25 (2001), 1213-1219.

[8] D. Tang, H.J. Park, S.P. Georgescu, S.M. Sebti, A.D. Hamilton and J.B. Galper, Simvastatin potentiates tumor necrosis factor alpha-mediated apoptosis of human vascular endothelial cells via the inhibition of the geranylgeranylation of RhoA, Life Sciences 79 (2006), 1484-1492.

[9] K. Fukui, S. Tamura, A. Wada, Y. Kamada, Y. Sawai, K. Imanaka, N. Hayashi, I. Shimomura and N. Hayashi, Expression and prognostic role of RhoAGTPases in hepatocellular carcinoma, Journal of Cancer Research and Clinical Oncology's 132 (2006), 627-633.

[10] A.P. Struckhoff, M.K. Rana and R.A. Worthylake, RhoA can lead the way in tumor cell invasion and metastasis, Frontiers in Bioscience 16 (2011), 1915-1926.

[11] X.R. Li, F. Ji, J. Ouyang, W. Wu, L.Y. Qian and K.Y. Yang, Overexpression of RhoA is associated with poor prognosis in hepatocellular carcinoma, European Journal of Surgical Oncology 32 (2006), 1130-1134.

[12] X.L. Deng, F. He, L.F. Yang, L.W. Wu and F. Yin, A novel mechanism of RhoA activation induced by tumor necrosis 
factor- $\alpha$ in mouse cerebral microvascular endothelial cells, Chinese Journal of Pathophysiology 27 (2011), 1210-1212.

[13] S. Lim, J. Ryu, J.A. Shin, M.J. Shin, Y.K. Ahn, J.J. Kim and K.H. Han, Tumor necrosis factor-alpha potentiates RhoAmediated monocyte transmigratory activity in vivo at a picomolar level, Arteriosclerosis Thrombosis and Vascular Biology 29 (2009), 2138-2145.

[14] T. Tuschl, Expanding small RNA interference, Nature Biotechnology 20 (2002), 446-448.

[15] Q.Q. Liu, K. Yin, S. Zhu, L. Zhang, P.E. Wen, C.L. Li, D.B. Zhang, M. Liu andG. Yan, Inhibition of C35 gene expression by small interfering RNA induces apoptosis of breast cancer cells, Bioscience Trends 4 (2010), 254-259.

[16] G. Yan, R. Duan, K. Yin, S. Zhu, Q. Liu, M. Gong, H. Wang, C. Sun, D. Pu, N. Tang and A.L. Huang, Inhibition of survivin expression to induce the apoptosis of hepatocarcinoma cells by adenovirus-mediated siRNA, Bioscience Trends 2 (2008), 88-93.

[17] T.C. He, S. Zhou, L.T. da Costa, J. Yu, K.W. Kinzler and B. Vogelstein, A simplified system for generating recombinant adenoviruses, Proceedings of the National Academy of Sciences of the United States of America 95 (1998), $2509-2514$

[18] M. Sioud, RNA interference: Mechanisms, technical challenges, and therapeutic opportunities, Methods in Molecular Biology 1218 (2015), 1-15.

[19] G. Li, H. Chang, Y.P. Zhai and W. Xu, Targeted silencing of inhibitors of apoptosis proteins with siRNAs: A potential anti-cancer strategy for hepatocellular carcinoma, Asian Pacific Journal of Cancer Prevention 14 (2013), 4943-4952.

[20] K. Yin, Q.Q. Liu, S. Zhu and G. Yan, Adenovirus-mediated siRNA inhibited survivin gene expression induces tumor cell apoptosis in nude mice, Bioscience Trends 2 (2008), 231-234.

[21] H. Wang, G. Zhao, X. Liu, A. Sui, K. Yang, R. Yao, Z. Wang and Q. Shi, Silencing of RhoA and RhoC expression by RNA interference suppresses human colorectal carcinoma growth in vivo, Journal of Experimental \& Clinical Cancer Research 29 (2010), 123.

[22] C.C. Wong, C.M. Wong, S.L. Au and I.O. Ng, RhoGTPases and Rho-effectors in hepatocellular carcinoma metastasis: ROCK N'Rhomove it, Liver International 30 (2010), 642-656.

[23] C.M. Wong, J.W. Yam, Y.P. Ching, T.O. Yau, T.H. Leung, D.Y. Jin and I.O. Ng, Rho GTPase-activating protein deleted in liver cancer suppresses cell proliferation and invasion in hepatocellular carcinoma, Cancer Research $\mathbf{6 5}$ (2005), 8861-8868.

[24] R. Rathinam, A. Berrier and S.K. Alahari, Role of Rho GTPases and their regulators in cancer progression, Frontiers in Bioscience-Landmark 16 (2011), 2561-2571.

[25] R.A. Cardone, A. Bellizzi, G. Busco, E.J. Weinman, M.E. Dell'Aquila, V. Casavola, A. Azzariti, A. Mangia, A. Paradiso and S.J. Reshkin, The NHERF1 PDZ2 domain regulates PKA-RhoA-p38-mediated NHE1 activation and invasion in breast tumor cells, Molecular Biology of The Cell 18 (2007), 1768-1780.

[26] F. Grise, A. Bidaud and V. Moreau, Rho GTPases in hepatocellular carcinoma, Biochimica et Biophysica Acta-General Subjects 1795 (2009), 137-151.

[27] L. Gou, W. Wang, A. Tong, Y. Yao, Y. Zhou, C. Yi and J. Yang, Proteomic identification of RhoA as a potential biomarker for proliferation and metastasis in hepatocellular carcinoma, Journal of Molecular Medicine (Berlin). 89 (2011), 817-827.

[28] A.Z. Hang, Q. Wang, Z. Han, W. Hu, L. Xi, Q. Gao, S. Wang, J. Zhou, G. Xu, L. Meng, G. Chen and D. Ma, Reduced expression of Snail decreases breast cancer cell motility by down-regulating the expression and inhibiting the activity of RhoAGTPase, Oncology Letters 6 (2013), 339-346.

[29] C.L. Williams, A new signaling paradigm to control the prenylation and trafficking of small GTPases, Cell Cycle 12 (2013), 2933-2934.

[30] X. Wang, H. He, Y. Lu, W. Ren, K.Y. Teng, C.L. Chiang, Z. Yang, B. Yu, S. Hsu, S.T. Jacob, K. Ghoshal and L.J. Lee, Indole-3-carbinol inhibits tumorigenicity of hepatocellular carcinoma cells via suppression of microRNA-21 and upregulation of phosphatase and tensin homolog, Biochimica et Biophysica Acta-general Subjects 1853 (2015), 244-253.

[31] Z. Li, X. Dong, Z. Wang, W. Liu, N. Deng, Y. Ding, L. Tang, T. Hla, R. Zeng, L. Li and D. Wu, Regulation of PTEN by Rho small GTPases, Nature Cell Biology 7 (2005), 399-404.

[32] S. Yang and H.M. Kim, The RhoA-ROCK-PTEN pathway as a molecular switch for anchorage dependent cell behavior, Biomaterials 33 (2012), 2902-2915.

[33] F. Fusella, R. Ferretti, D. Recupero, S. Rocca, A. Di Savino, G. Tornillo, L. Silengo, E. Turco, S. Cabodi, P. Provero, P.P. Pandolfi, A. Sapino, G. Tarone and M. Brancaccio, Morgana acts as a proto-oncogene through inhibition of a ROCK-PTEN pathway, Journal of Patholoy and Bacteriology 234 (2014), 152-163.

[34] K. Goto, Y. Chiba, K. Matsusue, Y. Hattori, Y. Maitani, H. Sakai, S. Kimura and M. Misawa, The proximal STAT6 and NF-kappaB sites are responsible for IL-13- and TNF-alpha-induced RhoA transcriptions in human bronchial smooth muscle cells, Pharmacological Research 61 (2010), 466-472.

[35] K. Goto, Y. Chiba, H. Sakai and M. Misawa, Tumor necrosis factor-alpha (TNF-alpha) induces up-regulation of RhoA 
via NF-kappaB activation in cultured human bronchial smooth muscle cells, Journal of Pharmacological Sciences 110 (2009), 437-444.

[36] E. Kakiashvili, P. Speight, F. Waheed, R. Seth, M. Lodyga, S. Tanimura, M. Kohno, O.D. Rotstein, A. Kapus and K. Szászi, GEF-H1 mediates tumor necrosis factor-alpha-induced Rho activation and myosin phosphorylation: Role in the regulation of tubular paracellular permeability, Journal of Biological Chemistry 284 (2009), 11454-11466.

[37] F. Balkwill, TNF-alpha in promotion and progression of cancer, cancer and Metastasis Reviews 25 (2006), 409-416. 